Virginia Commonwealth University VCU Scholars Compass

\title{
Pioglitazone, Vitamin E, or Placebo for Nonalcoholic Steatohepatitis
}

\author{
Arun J. Sanyal \\ Naga Chalasani \\ Indiana University \\ Kris V. Kowdley \\ Virginia Mason Medical Center \\ See next page for additional authors
}

VirginiaCommonwealth University, asanyal@mcvh-vcu.edu

Follow this and additional works at: http://scholarscompass.vcu.edu/intmed_pubs

Part of the Medicine and Health Sciences Commons

From The New England Journal of Medicine, Sanyal, A. J., Chalasani, N., Kowdley, K. V. et al., Pioglitazone, Vitamin E, or Placebo for Nonalcoholic Steatohepatitis, Vol. 362, Page 1675, Copyright (C) 2010 Massachusetts Medical Society.

\section{Downloaded from}

http://scholarscompass.vcu.edu/intmed_pubs/42

\footnotetext{
This Article is brought to you for free and open access by the Dept. of Internal Medicine at VCU Scholars Compass. It has been accepted for inclusion in Internal Medicine Publications by an authorized administrator of VCU Scholars Compass. For more information, please contact libcompass@vcu.edu.
} 


\section{Authors}

Arun J. Sanyal, Naga Chalasani, Kris V. Kowdley, Arthur McCollough, Anna Mae Diehl, Nathan M. Bass, Brent A. Neuschwander-Tetri, Joel E. Lavine, James Tonascia, Aynur Unalp, Mark Van Natta, Jeanne Clark, Elizabeth M. Brunt, David E. Kleiner, Jay H. Hoofnagle, and Patricia R. Robuck 


\section{Pioglitazone, Vitamin E, or Placebo for Nonalcoholic Steatohepatitis}

\author{
Arun J. Sanyal, M.D., Naga Chalasani, M.B., B.S., Kris V. Kowdley, M.D., \\ Arthur McCullough, M.D., Anna Mae Diehl, M.D., Nathan M. Bass, M.D., Ph.D., \\ Brent A. Neuschwander-Tetri, M.D., Joel E. Lavine, M.D., Ph.D., \\ James Tonascia, Ph.D., Aynur Unalp, M.D., Ph.D., Mark Van Natta, M.H.S., \\ Jeanne Clark, M.D., M.P.H., Elizabeth M. Brunt, M.D., \\ David E. Kleiner, M.D., Ph.D., Jay H. Hoofnagle, M.D., \\ and Patricia R. Robuck, Ph.D., M.P.H., for the NASH CRN*
}

ABSTRACT

\section{BACKGROUND}

Nonalcoholic steatohepatitis is a common liver disease that can progress to cirrhosis. Currently, there is no established treatment for this disease.

\section{METHODS}

We randomly assigned 247 adults with nonalcoholic steatohepatitis and without diabetes to receive pioglitazone at a dose of $30 \mathrm{mg}$ daily ( 80 subjects), vitamin $\mathrm{E}$ at a dose of 800 IU daily ( 84 subjects), or placebo ( 83 subjects), for 96 weeks. The primary outcome was an improvement in histologic features of nonalcoholic steatohepatitis, as assessed with the use of a composite of standardized scores for steatosis, lobular inflammation, hepatocellular ballooning, and fibrosis. Given the two planned primary comparisons, $\mathrm{P}$ values of less than 0.025 were considered to indicate statistical significance.

\section{RESULTS}

Vitamin E therapy, as compared with placebo, was associated with a significantly higher rate of improvement in nonalcoholic steatohepatitis ( $43 \%$ vs. $19 \%, \mathrm{P}=0.001)$, but the difference in the rate of improvement with pioglitazone as compared with placebo was not significant (34\% and 19\%, respectively; $\mathrm{P}=0.04$ ). Serum alanine and aspartate aminotransferase levels were reduced with vitamin $\mathrm{E}$ and with pioglitazone, as compared with placebo ( $\mathrm{P}<0.001$ for both comparisons), and both agents were associated with reductions in hepatic steatosis $(\mathrm{P}=0.005$ for vitamin $\mathrm{E}$ and $\mathrm{P}<0.001$ for pioglitazone) and lobular inflammation $(\mathrm{P}=0.02$ for vitamin $\mathrm{E}$ and $\mathrm{P}=0.004$ for pioglitazone) but not with improvement in fibrosis scores $(\mathrm{P}=0.24$ for vitamin $\mathrm{E}$ and $\mathrm{P}=0.12$ for pioglitazone). Subjects who received pioglitazone gained more weight than did those who received vitamin $\mathrm{E}$ or placebo; the rates of other side effects were similar among the three groups.

\section{CONCLUSIONS}

Vitamin E was superior to placebo for the treatment of nonalcoholic steatohepatitis in adults without diabetes. There was no benefit of pioglitazone over placebo for the primary outcome; however, significant benefits of pioglitazone were observed for some of the secondary outcomes. (ClinicalTrials.gov number, NCT00063622.)
From Virginia Commonwealth University, Richmond (A.J.S.); Indiana University, Indianapolis (N.C.); Virginia Mason Medical Center, Seattle (K.V.K.); Case Western Reserve University, Cleveland (A.M.); Duke University, Durham, NC (A.M.D.); University of California San Francisco, San Francisco (N.M.B.); Saint Louis University (B.A.N.-T.) and Washington University (E.M.B.) - both in St. Louis; University of California San Diego, San Diego (J.E.L.); Johns Hopkins University, Baltimore (J.T., A.U., M.V.N., J.C.); and the National Cancer Institute (D.E.K.) and National Institute of Diabetes and Digestive and Kidney Diseases (J.H.H., P.R.R.) - both in Bethesda, MD. Address reprint requests to Dr. Sanyal at MCV Box 980342, Richmond, VA 23298-0342, or at asanyal@mcvh-vcu.edu.

*The members of the Nonalcoholic Steatohepatitis Clinical Research Network (NASH CRN) are listed in the Appendix.

This article (10.1056/NEJMoa0907929) was published on April 28, 2010, at NEJM.org.

N Engl J Med 2010;362:1675-85.

Copyright $(2010$ Massachusetts Medical Society. 
N

ONALCOHOLIC STEATOHEPATITIS IS A common liver disease that is characterized histologically by hepatic steatosis, lobular inflammation, and hepatocellular ballooning $^{1,2}$; it can progress to cirrhosis in up to $15 \%$ of patients. ${ }^{3,4}$ There is currently no therapy that is of proven benefit for nonalcoholic steatohepatitis. The disease is closely associated with insulin resistance and features of the metabolic syndrome such as obesity, hypertriglyceridemia, and type 2 diabetes. ${ }^{5,6}$ In addition to insulin resistance, oxidative stress has been implicated as a key factor contributing to hepatic injury in patients with nonalcoholic steatohepatitis. ${ }^{6,7}$ Thus, both insulin resistance and oxidative stress are attractive targets for therapy in patients with this disease.

Several pilot studies have provided evidence that insulin sensitizers such as thiazolidinediones and antioxidants such as vitamin E improve clinical and histologic features of nonalcoholic steatohepatitis..$^{8-14}$ The medical evidence of a benefit, however, is limited, because these studies had small samples and were performed at single centers. Moreover, a recent multicenter trial showed a reduction in hepatic steatosis but no improvement in markers of cell injury after a year of rosiglitazone therapy. ${ }^{15}$ The value of these drugs remains uncertain.

The Nonalcoholic Steatohepatitis Clinical Research Network (NASH CRN) was established by the National Institute of Diabetes and Digestive and Kidney Diseases (NIDDK) to address unmet research needs in the field. As a part of this mandate, the NASH CRN conducted the Pioglitazone versus Vitamin E versus Placebo for the Treatment of Nondiabetic Patients with Nonalcoholic Steatohepatitis (PIVENS) trial, a phase 3 , multicenter, randomized, placebo-controlled, double-blind clinical trial of pioglitazone or vitamin $\mathrm{E}$ for the treatment of adults without diabetes who had biopsy-confirmed nonalcoholic steatohepatitis.

\section{METHODS}

\section{STUDY OVERSIGHT}

The PIVENS protocol and consent forms were designed by a subcommittee and approved by the steering committee of the CRN (see the Appendix). The study was approved by the review board at each participating center, and all subjects gave written informed consent. The study was conducted by site investigators, and data were gath- ered by specifically trained personnel. The data were analyzed at the data coordinating center and were reviewed by both the investigators and an independent data and safety monitoring board. The manuscript was written by a CRN subcommittee and approved by the members of the steering committee, who assume responsibility for the integrity of the data and the overall content of the manuscript.

\section{STUDY DESIGN}

The trial design and rationale for the PIVENS trial have been described previously. ${ }^{16}$ This trial focused on adults without diabetes who had nonalcoholic steatohepatitis. Adults with diabetes were excluded because it was unclear whether they would have the same responses to therapy as would adults without diabetes and because it was possible that changes in antidiabetic therapy might confound the analysis of data both from subjects with diabetes and from those without diabetes. All subjects underwent a liver biopsy within 6 months before randomization.

Steatohepatitis was categorized as absent, possible, or definite. ${ }^{17}$ Disease activity was assessed with the use of the nonalcoholic fatty liver disease activity score, which is based on a standardized grading system for steatosis (on a scale of 0 to 3 ), lobular inflammation (on a scale of 0 to 3), and hepatocellular ballooning (on a scale of 0 to 2), with higher scores indicating increasing severity). ${ }^{17}$ The specific inclusion criteria were definite or possible steatohepatitis with an activity score of 5 or more, or definite steatohepatitis (confirmed by two pathologists) with an activity score of 4 . A score of at least 1 for hepatocellular ballooning was required in all cases. Although eligibility was determined by the pathologist at each site from an assessment of tissue sections prepared locally, the final analysis was based on review by a pathology committee of deeper cuts, prepared centrally, from the biopsy specimens obtained at baseline and at the end of treatment. Members of the pathology committee who performed the final analysis were unaware of the study-group assignments.

Exclusion criteria were alcohol consumption of more than $20 \mathrm{~g}$ per day in the case of women and more than $30 \mathrm{~g}$ per day in the case of men for at least 3 consecutive months during the previous 5 years, as assessed with the use of the Lifetime Drinking History questionnaire of Skinner et al. ${ }^{18}$ and the self-administered Alcohol Use and Disor- 
ders Identification Test (AUDIT). ${ }^{19}$ Subjects were also excluded if they had cirrhosis, hepatitis $\mathrm{C}$ or other liver diseases, or heart failure (New York Heart Association class II to IV), or if they were receiving drugs known to cause steatohepatitis.

Both at the initial evaluation and at the completion of treatment 96 weeks later, subjects underwent an assessment of body weight, height, and waist and hip circumferences, and blood samples were obtained for routine biochemical tests and assessment of fasting levels of lipids, glucose, and insulin. Body composition was assessed with the use of dual-energy x-ray absorptiometry. The Medical Outcomes Study 36-Item Short-Form Health Survey (SF-36) was administered for the assessment of quality of life.

\section{RANDOMIZATION AND TREATMENT GROUPS}

Subjects who met all eligibility criteria and provided written informed consent were randomly assigned to one of three groups for 96 weeks of study treatment: a group receiving pioglitazone (at a dose of $30 \mathrm{mg}$ once daily) and a vitamin E-like placebo (once daily), a group receiving vitamin E (800 IU, natural form, once daily) and a pioglitazone-like placebo (once daily); or a group receiving a pioglitazone-like placebo (once daily) and a vitamin E-like placebo (once daily). The 30-mg pioglitazone tablets (Actos) and similarappearing placebo tablets were provided by Takeda Pharmaceuticals North America under a collaborative research and development agreement with the NIDDK. The 800-IU liquid soft-gelatin capsules of RRR- $\alpha$-tocopherol (formerly known as $\mathrm{d}$ - $\alpha$-tocopherol) vitamin $\mathrm{E}$ (Nature Made) and similar-appearing placebo soft-gelatin capsules were provided by Pharmavite under a clinical trial agreement with the NIDDK. Both Takeda and Pharmavite provided comments regarding the study design but were not involved in the writing of the protocol, the conduct of the trial, the decision-making with respect to the trial, or the analysis of the data. Both companies were shown a draft of the manuscript 30 days before it was submitted for publication but were not involved in the preparation of the manuscript. The doses were selected on the basis of previous phase 2 studies. ${ }^{9,13}$

\section{FOLLOW-UP VISITS}

After randomization, subjects were followed according to a predetermined schedule for assess- ment of the safety and tolerability of the study drugs. Subjects were discouraged from adding other drugs that are used for nonalcoholic steatohepatitis to their regimen. All subjects were given a standardized set of pragmatic recommendations about lifestyle changes and diet. A liver biopsy was performed 96 weeks after randomization, after which the study drugs were discontinued. Subjects were then followed for an additional 24 weeks.

\section{PRIMARY AND SECONDARY OUTCOMES}

The primary outcome was an improvement in histologic findings, which required an improvement by 1 or more points in the hepatocellular ballooning score; no increase in the fibrosis score; and either a decrease in the activity score for nonalcoholic fatty liver disease to a score of 3 or less or a decrease in the activity score of at least 2 points, with at least a 1-point decrease in either the lobular inflammation or steatosis score.

Secondary outcomes included changes in the overall activity score for nonalcoholic fatty liver disease, as well as in individual component scores for steatosis, lobular inflammation, hepatocellular ballooning, and fibrosis, and changes in serum aminotransferase levels, anthropometric measures, and insulin resistance and lipid profiles. An additional secondary outcome was the change in health-related quality of life from baseline to the end of treatment, as assessed with the use of the SF-36.

\section{STATISTICAL ANALYSIS}

The primary analysis was an intention-to-treat analysis in which the proportions of subjects in each active-treatment group (pioglitazone and vitamin E) in whom there was improvement in nonalcoholic steatohepatitis were compared with the proportion of subjects in the placebo group in whom there was improvement. Comparisons were made with the use of the Mantel-Haenszel chisquare test, stratified according to clinical site. Subjects who did not undergo an end-of-treatment biopsy were classified as not having had improvement. Given the fact that there were two planned primary comparisons, Bonferroni-adjusted P values of less than 0.025 were considered to indicate statistical significance. The number of subjects who would need to be treated to achieve histologic improvement in one patient was calculated for the vitamin $\mathrm{E}$ and pioglitazone treatments. We analyzed secondary outcomes with the use of 
Fisher's exact test for binary outcomes and analysis-of-covariance models for continuous outcomes, regressing change from baseline to 96 weeks on treatment group and baseline value of the outcome variable in order to adjust comparisons of mean changes in secondary outcomes for the baseline value of the outcome variable.

The planned sample size was 240 subjects, with equal assignment to each of the three study groups (80 per group). We estimated that with this sample size, the study would have $90 \%$ power to detect an absolute difference in the rate of improvement in nonalcoholic steatohepatitis of 26 percentage points, with a two-sided type 1 error of $0.025 .{ }^{16}$ An independent data and safety monitoring board appointed by the NIDDK monitored the results semiannually; O'Brien-Fleming statistical stopping guidelines were used, with one interim analysis for efficacy performed midway through the trial.

RESULTS

\section{STUDY SUBJECTS}

Subjects were enrolled in the trial between January 2005 and January 2007 and were treated and followed for 96 weeks (Fig. 1). Of the 247 subjects who underwent randomization, 83 were assigned to receive placebo, 84 were assigned to receive vitamin $\mathrm{E}$, and 80 were assigned to receive pioglitazone. The three groups were well matched with respect to demographic characteristics, clinical and laboratory data, and activity scores for nonalcoholic fatty liver disease (Table 1). However, according to the planned, blinded, central analysis of liver-biopsy specimens, $17 \%$ of the subjects in the placebo group, $18 \%$ of those in the vitamin E group, and $28 \%$ of those in the pioglitazone group did not have hepatocellular ballooning on assessment of their initial biopsy specimen.

\section{PRIMARY OUTCOME}

A total of $90 \%$ of the subjects underwent an endof-treatment liver biopsy at 96 weeks. Vitamin E therapy, as compared with placebo, was associated with a significantly higher rate of improvement in nonalcoholic steatohepatitis $(43 \%$ vs. $19 \%$, $\mathrm{P}=0.001$; number needed to treat, 4.2 ), but the comparison of pioglitazone therapy with placebo did not reach the prespecified 0.025 level of significance for the primary outcome (34\% vs. $19 \%$, $\mathrm{P}=0.04$; number needed to treat, 6.9) (Table 2).
Given the subjectivity of histologic analysis, several sensitivity analyses were performed. If a finding of no worsening (rather than improvement) of hepatocellular ballooning was used as a criterion in the outcome measure, both active drugs were significantly associated with an improvement in histologic features of nonalcoholic steatohepatitis, as compared with placebo (placebo vs. vitamin E: $25 \%$ vs. $51 \%$ improvement, $\mathrm{P}<0.001$; placebo vs. pioglitazone: $25 \%$ vs. $48 \%$, $\mathrm{P}=0.003)$. When subjects who did not have hepatocellular ballooning initially, as assessed by central review, were excluded from the analyses, both active drug groups were associated with a significant improvement in histologic findings (placebo vs. vitamin E: $23 \%$ vs. $52 \%$ improvement, $\mathrm{P}<0.001$; placebo vs. pioglitazone: $23 \%$ vs. $47 \%, \mathrm{P}=0.002$ ). An analysis of baseline factors to identify characteristics associated with a histologic response to either active treatment did not reveal any significant findings, and the data were consistent across all subgroups that were analyzed (Table 10 in the Supplementary Appendix, available with the full text of this article at NEJM.org).

\section{SECONDARY OUTCOMES}

\section{Histologic Features}

As compared with the placebo group, both active-treatment groups had a significant reduction in steatosis, lobular inflammation (Fig. 1 in the Supplementary Appendix), and the activity score for nonalcoholic fatty liver disease (Table 2). Although scores for hepatocellular ballooning were improved with vitamin $\mathrm{E}$ and with pioglitazone, only the improvement with vitamin $\mathrm{E}$ was significant $(P=0.01)$. Fibrosis scores were not significantly improved with either active treatment. Steatohepatitis resolved in a greater proportion of subjects receiving either vitamin $\mathrm{E}$ or pioglitazone than in those receiving placebo, but the difference was significant only for those receiving pioglitazone $(\mathrm{P}=0.001)$ (Table 2).

\section{Serum Enzyme Levels and Liver Test Results}

There was an early and highly significant decrease in mean aspartate aminotransferase and alanine aminotransferase levels among subjects receiving vitamin $\mathrm{E}$ and among subjects receiving pioglitazone (Fig. 2). These changes occurred in the first 24 weeks and were sustained throughout the period in which the subjects were receiving treatment. Serum concentrations of alkaline phospha- 


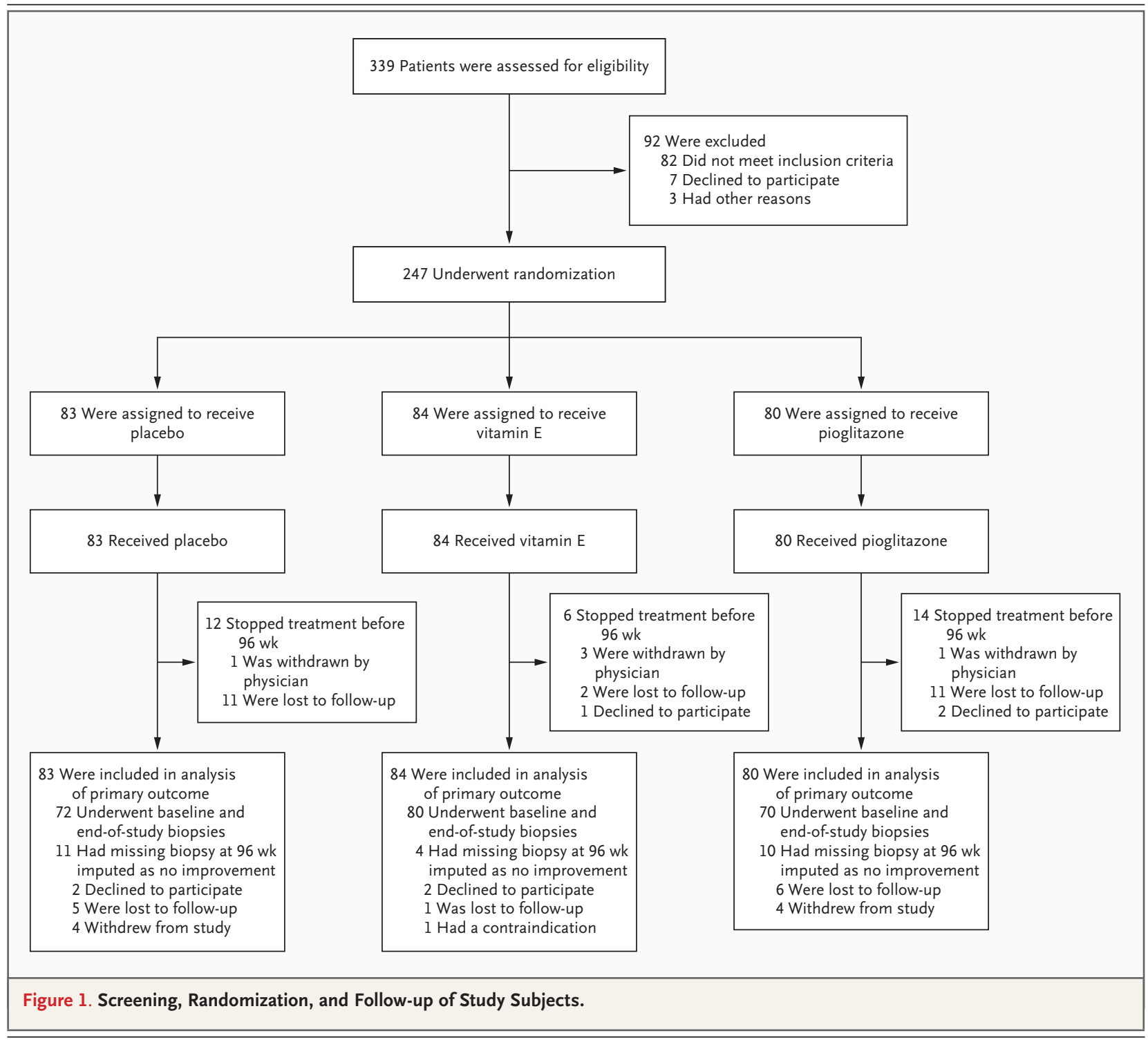

tase and $\gamma$-glutamyl transpeptidase also improved in the two active-drug groups, but bilirubin levels did not change significantly.

Body Weight, Insulin Resistance, and Quality of Life There was a significant improvement in insulin resistance only in the pioglitazone group, but that group was also the only group that had a significant mean weight gain (a mean increase of $4.7 \mathrm{~kg}$ at week 96, $\mathrm{P}<0.001$ for the comparison with placebo) (Table 3). These changes were noted by week 24 , and the weight gain progressed over the course of the study. Although insulin resistance returned to the baseline value after discontinuation of pioglitazone, the associated weight gain was not re- versed. Changes in quality of life, as assessed by the scores for both the mental and physical components of the SF-36, did not differ significantly between the vitamin $\mathrm{E}$ group or the pioglitazone group and the placebo group (Table 3).

\section{Adverse Events}

With the exception of weight gain, the overall distribution of individual adverse events did not differ significantly across the study groups. There were 19 severe adverse events - 10 in the placebo group, 7 in the vitamin E group, and 2 in the pioglitazone group (see Table 3 in the Supplementary Appendix for details). A similar number of cardiovascular events occurred among subjects 


\begin{tabular}{|c|c|c|c|c|}
\hline Characteristic & Placebo $(\mathrm{N}=83)$ & Vitamin $E(N=84)$ & Pioglitazone $(\mathrm{N}=80)$ & Total $(\mathrm{N}=\mathbf{2 4 7})$ \\
\hline \multicolumn{5}{|l|}{ Demographic factors } \\
\hline Age (yr) & $45.4 \pm 11.2$ & $46.6 \pm 12.1$ & $47.0 \pm 12.6$ & $46.3 \pm 11.9$ \\
\hline Female sex (\%) & 58 & 62 & 59 & 60 \\
\hline \multicolumn{5}{|l|}{ Race or ethnic group (\%) † } \\
\hline Hispanic & 7 & 19 & 19 & 15 \\
\hline Nonwhite & 11 & 15 & 8 & 12 \\
\hline \multicolumn{5}{|l|}{ Quality of life } \\
\hline SF-36, physical component & $47 \pm 11$ & $49 \pm 10$ & $49 \pm 9$ & $48 \pm 10$ \\
\hline SF-36, mental component & $47 \pm 12$ & $49 \pm 10$ & $49 \pm 8$ & $48 \pm 10$ \\
\hline \multicolumn{5}{|l|}{ Serum biochemical levels } \\
\hline Alanine aminotransferase (U/liter) & $81 \pm 48$ & $86 \pm 52$ & $82 \pm 45$ & $83 \pm 49$ \\
\hline Aspartate aminotransferase (U/liter) & $55 \pm 30$ & $59 \pm 33$ & $54 \pm 26$ & $56 \pm 30$ \\
\hline$\gamma$-Glutamyltransferase (U/liter) & $69 \pm 79$ & $56 \pm 42$ & $60 \pm 63$ & $61 \pm 63$ \\
\hline Alkaline phosphatase (U/liter) & $82 \pm 25$ & $77 \pm 25$ & $86 \pm 39$ & $81 \pm 30$ \\
\hline Total bilirubin (mg/dl) & $0.76 \pm 0.35$ & $0.75 \pm 0.38$ & $0.77 \pm 0.38$ & $0.76 \pm 0.37$ \\
\hline \multicolumn{5}{|l|}{ Lipids } \\
\hline Triglycerides (mg/dl) & $165 \pm 89$ & $166 \pm 104$ & $162 \pm 84$ & $165 \pm 93$ \\
\hline \multicolumn{5}{|l|}{ Cholesterol (mg/dl) } \\
\hline Total & $199 \pm 40$ & $195 \pm 40$ & $195 \pm 37$ & $196 \pm 39$ \\
\hline High-density lipoprotein & $43 \pm 11$ & $44 \pm 13$ & $45 \pm 12$ & $44 \pm 12$ \\
\hline Low-density lipoprotein & $125 \pm 35$ & $119 \pm 35$ & $120 \pm 31$ & $122 \pm 34$ \\
\hline \multicolumn{5}{|l|}{ Metabolic factors } \\
\hline Fasting serum glucose (mg/dl) & $95 \pm 14$ & $95 \pm 14$ & $92 \pm 12$ & $94 \pm 13$ \\
\hline Insulin resistance $\mathbb{Z}$ & $5.5 \pm 5.1$ & $5.2 \pm 4.0$ & $5.0 \pm 3.8$ & $5.2 \pm 4.3$ \\
\hline Weight (kg) & $99 \pm 21$ & $94 \pm 24$ & $97 \pm 23$ & $97 \pm 23$ \\
\hline Body-mass index 9 & $35 \pm 7$ & $34 \pm 7$ & $34 \pm 6$ & $34 \pm 7$ \\
\hline Waist circumference $(\mathrm{cm})$ & $109 \pm 14$ & $107 \pm 15$ & $108 \pm 14$ & $108 \pm 14$ \\
\hline Body composition (\% fat) & $40 \pm 9$ & $39 \pm 9$ & $40 \pm 8$ & $39 \pm 9$ \\
\hline \multicolumn{5}{|l|}{ Liver histologic findings $\|$} \\
\hline Total NAFLD activity score & $4.8 \pm 1.4$ & $5.1 \pm 1.4$ & $5.0 \pm 1.4$ & $4.9 \pm 1.4$ \\
\hline Steatosis & $1.9 \pm 0.8$ & $1.9 \pm 0.9$ & $2.0 \pm 0.8$ & $1.9 \pm 0.8$ \\
\hline Lobular inflammation & $1.6 \pm .0 .7$ & $1.8 \pm 0.7$ & $1.8 \pm 0.7$ & $1.7 \pm 0.7$ \\
\hline Hepatocellular ballooning & $1.3 \pm 0.7$ & $1.3 \pm 0.8$ & $1.1 \pm 0.8$ & $1.3 \pm 0.8$ \\
\hline Fibrosis stage*** & $1.6 \pm 1.1$ & $1.5 \pm 1.0$ & $1.4 \pm 0.9$ & $1.5 \pm 1.0$ \\
\hline Absence of ballooning on central review (\%) & 17 & 18 & 28 & 21 \\
\hline
\end{tabular}

* Plus-minus values are means \pm SD. To convert the values for bilirubin to micromoles per liter, multiply by 17.1. To convert the values for triglycerides to millimoles per liter, multiply by 0.01129 . To convert the values for cholesterol to millimoles per liter, multiply by 0.02586 . To convert the values for glucose to millimoles per liter, multiply by 0.05551 .

$\dagger$ Race or ethnic group was self-reported.

Scores on the Medical Outcomes Study 36-Item Short-Form Health Survey (SF-36) were standardized to the 1998 U.S. general population with a mean $( \pm S D)$ of $50 \pm 10$.

$\int$ The homeostasis model assessment for insulin resistance (HOMA-IR) was used to calculate insulin resistance, according to the following formula: (milligrams of glucose per deciliter $\times$ microunits of insulin per milliliter) $\div 405$. Higher numbers indicate greater insulin resistance.

9 The body-mass index is the weight in kilograms divided by the square of the height in meters.

|| Total nonalcoholic fatty liver disease (NAFLD) activity was assessed on a scale of 0 to 8 , with higher scores indicating more severe disease; the components of this measure include steatosis (assessed on a scale of 0 to 3), lobular inflammation (assessed on a scale of 0 to 3 ), and hepatocellular ballooning (assessed on a scale of 0 to 2 ).

* $*$ Fibrosis was assessed on a scale of 0 to 4 , with higher scores indicating more severe fibrosis. 


\begin{tabular}{|c|c|c|c|c|c|}
\hline \multirow[t]{2}{*}{ Variable } & \multirow[t]{2}{*}{ Placebo } & \multirow[t]{2}{*}{ Vitamin E } & \multirow[t]{2}{*}{ Pioglitazone } & \multicolumn{2}{|c|}{ P Value* } \\
\hline & & & & $\begin{array}{l}\text { Vitamin E } \\
\text { vs. Placebo }\end{array}$ & $\begin{array}{l}\text { Pioglitazone } \\
\text { vs. Placebo }\end{array}$ \\
\hline \multicolumn{6}{|l|}{ Primary outcome } \\
\hline No. of subjects randomly assigned & 83 & 84 & 80 & & \\
\hline Subjects with improvement (\%) & 19 & 43 & 34 & 0.001 & 0.04 \\
\hline \multicolumn{6}{|l|}{ Changes from baseline in histologic features } \\
\hline $\begin{array}{l}\text { No. of subjects with biopsy specimens at baseline } \\
\text { and } 96 \mathrm{wk}\end{array}$ & 72 & 80 & 70 & & \\
\hline \multicolumn{6}{|l|}{ Steatosis } \\
\hline Subjects with improvement (\%) & 31 & 54 & 69 & 0.005 & $<0.001$ \\
\hline Mean change in score & -0.1 & -0.7 & -0.8 & $<0.001$ & $<0.001$ \\
\hline \multicolumn{6}{|l|}{ Lobular inflammation } \\
\hline Subjects with improvement (\%) & 35 & 54 & 60 & 0.02 & 0.004 \\
\hline Mean change in score & -0.2 & -0.6 & -0.7 & 0.008 & $<0.001$ \\
\hline \multicolumn{6}{|l|}{ Hepatocellular ballooning } \\
\hline Subjects with improvement (\%) & 29 & 50 & 44 & 0.01 & 0.08 \\
\hline Mean change in score & -0.2 & -0.5 & -0.4 & 0.03 & 0.01 \\
\hline Total NAFLD activity score (mean change) & -0.5 & -1.9 & -1.9 & $<0.001$ & $<0.001$ \\
\hline \multicolumn{6}{|l|}{ Fibrosisł } \\
\hline Subjects with improvement (\%) & 31 & 41 & 44 & 0.24 & 0.12 \\
\hline Mean change in score & -0.1 & -0.3 & -0.4 & 0.19 & 0.10 \\
\hline $\begin{array}{l}\text { Resolution of definite nonalcoholic steatohepatitis } \\
\text { (\% of subjects) }\end{array}$ & 21 & 36 & 47 & 0.05 & 0.001 \\
\hline
\end{tabular}

* P values were calculated with the use of the Mantel-Haenszel chi-square test, stratified according to clinic, for the primary outcome; Fisher's exact test for the binary secondary outcomes; and analysis-of-covariance models, regressing change from baseline to 96 weeks on treatment group and baseline value of the outcome, for secondary outcome scores.

The primary outcome was an improvement in histologic findings, which required improvement by 1 or more points in the hepatocellular ballooning score; no increase in the fibrosis score; and either a decrease in the activity score for nonalcoholic fatty liver disease to a score of 3 points or less or a decrease in the activity score of at least 2 points, with at least a 1-point decrease in either the lobular inflammation or steatosis score. A total of 11 subjects in the placebo group, 4 in the vitamin E group, and 10 in the pioglitazone group had missing histologic data at week 96 , and the results for these subjects were imputed as a lack of improvement. The NAFLD activity score was assessed on a scale of 0 to 8, with higher scores indicating more severe disease; the components of this measure include steatosis (assessed on a scale of 0 to 3 ), lobular inflammation (assessed on a scale of 0 to 3 ), and hepatocellular ballooning (assessed on a scale of 0 to 2 ).

$\$$ Fibrosis was assessed on a scale of 0 to 4 , with higher scores indicating more severe fibrosis.

taking placebo (12 events), those taking vitamin $\mathrm{E}$ (12), and those taking pioglitazone (10). No cases of congestive heart failure were reported. The frequency of bone fractures was also similar across study groups: five in the placebo group, three in the vitamin E group, and three in the pioglitazone group. During the 96 weeks of the study, diabetes developed in four subjects who were receiving vitamin $\mathrm{E}$ but in none of the subjects who were receiving placebo or pioglitazone $(\mathrm{P}=0.12$ for the comparison of vitamin $\mathrm{E}$ with placebo). Symptoms and signs of cirrhosis (thrombocytope- nia, anasarca, and hyperbilirubinemia) developed in one patient in the vitamin E group who had had bridging hepatic fibrosis at entry, and that patient died of sepsis. No cases of serious hepatotoxicity requiring permanent discontinuation of the study drug were reported.

\section{DISCUSSION}

The assessment of therapeutic agents for nonalcoholic steatohepatitis is a complex process. Because there are no validated biomarkers of response 


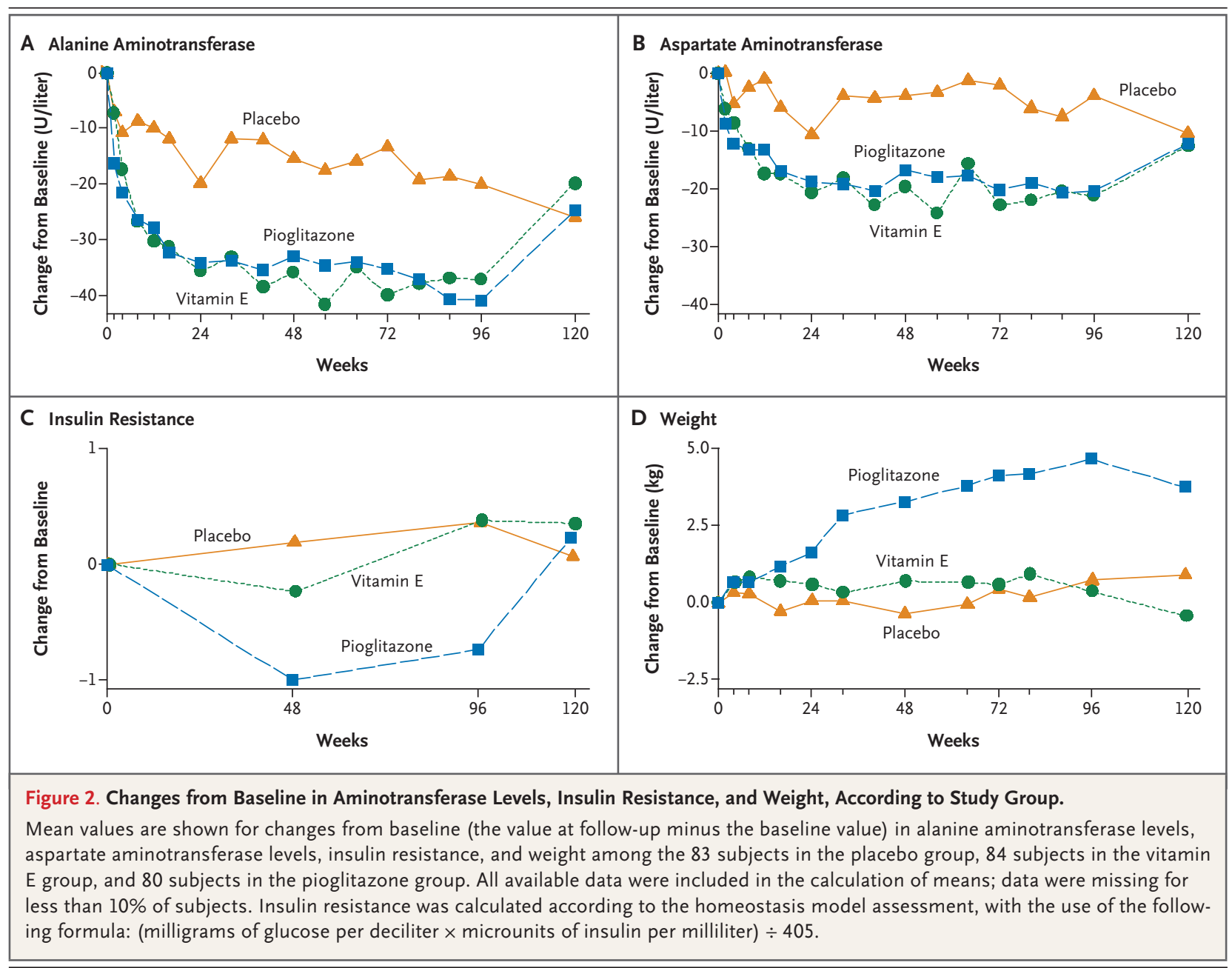

to treatment, one must rely on histologic assessment of a liver-biopsy specimen for this purpose. The activity score for nonalcoholic fatty liver disease quantifies the severity of steatosis, hepatocellular ballooning, and inflammation - the key histologic components of the disease. ${ }^{17}$ A decrease in their severity occurs with amelioration of the disease; however, the severity of these components (especially hepatic steatosis) may also decrease with progression of fibrosis to cirrhosis. ${ }^{20,21}$

To develop an outcome that was both quantifiable and clinically relevant, the requirements of an improvement in ballooning and no worsening of fibrosis were added to the requirement of a decrease in the nonalcoholic fatty liver disease activity score for the primary outcome. This makes it likely that the observed improvements with vitamin E are both statistically and clinically significant. These improvements are also concordant with those in previous small, pilot trials. ${ }^{12,13}$ These data cannot, however, be generalized to patients with diabetes or to those with cirrhosis, and additional clinical trials both to confirm these findings and to determine the generalizability of the data are warranted.

Although pioglitazone did not meet the prespecified significance level for the primary outcome, it was associated with highly significant reductions in steatosis, inflammation, and hepatocellular ballooning, as well as with improvements in insulin resistance and liver-enzyme levels (Table 2 and Fig. 2). It also led to the resolution of steatohepatitis in a significant proportion of subjects. One possible reason for the failure to achieve the primary outcome with pioglitazone therapy, even though it significantly improved individual features of nonalcoholic steatohepatitis, is that more subjects in the pioglitazone group than in the vitamin $\mathrm{E}$ and placebo groups were classified as not having had ballooning, on the basis of the planned central review of deeper cuts of baseline biopsy specimens, and a reduction in 


\begin{tabular}{|c|c|c|c|c|c|}
\hline \multirow[t]{2}{*}{ Variable } & \multicolumn{3}{|c|}{ Change from Baseline } & \multicolumn{2}{|c|}{ P Value'̀ } \\
\hline & $\begin{array}{l}\text { Placebo } \\
(\mathrm{N}=74)\end{array}$ & $\begin{array}{l}\text { Vitamin E } \\
(N=78)\end{array}$ & $\begin{array}{l}\text { Pioglitazone } \\
(N=70)\end{array}$ & $\begin{array}{l}\text { Vitamin E } \\
\text { vs. Placebo }\end{array}$ & $\begin{array}{l}\text { Pioglitazone } \\
\text { vs. Placebo }\end{array}$ \\
\hline \multicolumn{6}{|l|}{ Serum enzymes and bilirubin } \\
\hline Alanine aminotransferase (U/liter) & -20.1 & -37.0 & -40.8 & 0.001 & $<0.001$ \\
\hline Aspartate aminotransferase (U/liter) & -3.8 & -21.3 & -20.4 & $<0.001$ & $<0.001$ \\
\hline$\gamma$-Glutamyltransferase (U/liter) & -4.0 & -14.0 & -21.1 & 0.003 & $<0.001$ \\
\hline Alkaline phosphatase (U/liter) & -3.8 & -9.3 & -12.0 & 0.008 & 0.004 \\
\hline Total bilirubin (mg/dl) & 0.06 & 0.04 & -0.04 & 0.56 & 0.07 \\
\hline \multicolumn{6}{|l|}{ Lipids } \\
\hline Triglycerides (mg/dl) & -6.7 & -0.6 & -19.8 & 0.45 & 0.16 \\
\hline \multicolumn{6}{|l|}{ Cholesterol (mg/dl) } \\
\hline Total & -9.6 & -13.6 & -11.4 & 0.25 & 0.50 \\
\hline High-density lipoprotein & -1.9 & -0.9 & 1.1 & 0.51 & 0.008 \\
\hline Low-density lipoprotein & -5.8 & -12.0 & -8.1 & 0.07 & 0.26 \\
\hline \multicolumn{6}{|l|}{ Metabolic factors } \\
\hline Fasting serum glucose (mg/dl) & 1.8 & 1.8 & -3.1 & 0.81 & 0.006 \\
\hline Insulin resistancet & 0.4 & 0.4 & -0.7 & 0.80 & 0.03 \\
\hline Weight $(\mathrm{kg})$ & 0.7 & 0.4 & 4.7 & 0.65 & $<0.001$ \\
\hline Body-mass index & 0.4 & 0.1 & 1.8 & 0.50 & $<0.001$ \\
\hline Waist circumference $(\mathrm{cm})$ & 0.2 & -0.4 & 3.3 & 0.53 & 0.06 \\
\hline Body composition (\% fat) & 0 & 0.4 & 2.7 & 0.50 & $<0.001$ \\
\hline \multicolumn{6}{|l|}{ Quality of life $\int$} \\
\hline SF-36 score, physical component & -0.3 & 0.4 & -0.9 & 0.45 & 0.93 \\
\hline SF-36 score, mental component & 0.4 & -0.5 & -1.9 & 0.76 & 0.23 \\
\hline
\end{tabular}

* The numbers of subjects listed for the three study groups are the numbers for whom data on alanine aminotransferase levels were available. The total numbers of subjects included in the analyses of other variables ranged from 212 to 223 . The change from baseline is the value at 96 weeks minus the baseline value. To convert the values for bilirubin to micromoles per liter, multiply by 17.1. To convert the values for triglycerides to millimoles per liter, multiply by 0.01129 . To convert the values for cholesterol to millimoles per liter, multiply by 0.02586 . To convert the values for glucose to millimoles per liter, multiply by 0.05551 .

$\dagger \mathrm{P}$ values were calculated with the use of analysis-of-covariance models, regressing change from baseline to 96 weeks on treatment group and baseline value of the outcome measure.

$\neq$ The homeostasis model assessment for insulin resistance (HOMA-IR) was used to calculate insulin resistance, according to the following formula: (milligrams of glucose per deciliter $\times$ microunits of insulin per milliliter) $\div 405$. Diabetes developed in four subjects assigned to the vitamin E group, and these subjects were not given an oral glucose-tolerance test at 96 weeks. Their HOMA-IR values at 96 weeks were imputed as the 95 th percentile value at baseline.

Analyses were performed with the use of ranks owing to asymmetry in the distribution of change in HOMA-IR values.

$\int$ Scores on the Medical Outcomes Study 36-Item Short-Form Health Survey (SF-36) were standardized to the 1998 U.S. general population with a mean $( \pm S D)$ of $50 \pm 10$.

ballooning was one of the criteria for the primary outcome. The discrepancies between the results of liver histologic assessments that were performed locally to determine eligibility and the results of assessments of deeper cuts of the same tissue block that were performed centrally to determine histologic baseline and outcome measures for use in the study analyses may have been due to interobserver variability, variable distribution of his- tologic findings in the different tissue sections, or a potential bias due to the unblinded nature of the local pathological review to determine eligibility. $22-24$

It is important not to overinterpret the data on adverse events in this trial because the study was not powered to test any safety-related hypotheses. The risk of bone fracture was not increased with pioglitazone ${ }^{25}$; however, the number of post- 
menopausal women included in the trial was too small to assess any potential effect. Although diabetes developed in subjects in the vitamin E group but not in subjects in the other two groups, only four subjects were affected, and this number is smaller than the number that would be expected in this patient population. Finally, cardiovascular events occurred with equal frequency in all three study groups, but, again, the trial was much too small to detect meaningful differences in the incidence of cardiovascular events.

Enthusiasm for the potential benefits of pioglitazone and vitamin $\mathrm{E}$ must be tempered by the finding that there was an improvement in histologic features in only $34 \%$ of the subjects who received pioglitazone and $43 \%$ of those who received vitamin $\mathrm{E}$, and steatohepatitis resolved in only $47 \%$ and $36 \%$ of the subjects in those two groups, respectively. Neither agent was associated with a significant improvement in the mean fibrosis score after 96 weeks of treatment. There was also no significant reduction in portal inflammation, which has been linked to advanced disease. ${ }^{4,26}$ Given the certainty of relapse after discontinuation of the drug, it is likely that whichever drug is prescribed for nonalcoholic steatohepatitis, it will need to be taken indefinitely. ${ }^{13,27}$ The weight gain among the subjects receiving pioglitazone - which did not resolve after discontinuation of the drug - also detracts from its long-term usefulness. The unknown long-term potential for adverse events with vitamin $\mathrm{E}$ and pioglitazone therapies must be factored into the decision about whether to use these agents. ${ }^{25,28,29}$

The decision about which specific therapy to use for the treatment of nonalcoholic steatohepatitis should include a consideration of both the efficacy and the toxic effects of the therapy as compared with those of other available therapies. Preliminary studies provide a rationale for the use of lifestyle intervention, bariatric surgery, phlebotomy, and a variety of drugs. However, the usefulness of each of these therapeutic options has not been validated in rigorously performed, randomized, controlled trials. Our study provides such evidence for vitamin $\mathrm{E}$ and pioglitazone; however, this trial was not designed to compare vitamin $\mathrm{E}$ with pioglitazone, and no conclusions can be drawn about their relative efficacy.

In summary, the results of this trial of pioglitazone and vitamin $\mathrm{E}$ for the treatment of nonalcoholic steatohepatitis in adults without diabetes showed that vitamin $\mathrm{E}$ was superior to placebo and suggested that pioglitazone may also have efficacy. Although only subjects who received vitamin $\mathrm{E}$ met the criteria for the prespecified primary outcome measure, subjects who received pioglitazone had significant improvement in other important histologic features of nonalcoholic steatohepatitis.

Supported by grants (U01DK61718, U01DK61728, U01DK61731, U01DK61732, U01DK61734, U01DK61737, U01DK61738, U01DK61730, and U01DK61713) from the National Institutes of Health (NIH), by the intramural program of the National Cancer Institute, and by NIH General Clinical Research Center grants or Clinical and Translational Science Awards (UL1RR024989, UL1RR024128, M01RR000750, UL1RR024131， M01RR000827, UL1RR025014, and M01RR000065). Additional funding was provided by Takeda Pharmaceuticals North America through a Cooperative Research and Development Agreement with the NIH. The vitamin E softgels and matching placebo were provided by Pharmavite through a Clinical Trial Agreement with the NIH.

Dr. Sanyal reports receiving consulting fees from Exalenz, Takeda Pharmaceuticals North America, Norgine, Astellas, and Amylin, grant support from Intercept Pharmaceuticals, Gilead, Roche, Salix Pharmaceuticals, Sanofi-Aventis, Otsuka, and Orphan Therapeutics, and royalties from UpToDate; Dr. Neuschwander-Tetri, consulting fees from Gilead, Astellas, Amylin, Centocor, Vertex, and Sanofi-Aventis and reimbursements for travel or accommodations expenses from Astellas and Gilead; Dr. Kowdley, consulting fees from Gilead, Novartis, Bristol-Myers Squibb, and Vertex, gifts from Roche, honoraria from Bristol-Myers Squibb, Schering-Plough (now Merck), and Roche, lecture fees from Bristol-Myers Squibb, grant support from Intercept, Schering-Plough (now Merck), and royalties from UpToDate; Dr. Diehl, consulting fees and reimbursements for travel or accommodations from CellszDirect and Vertex, and grant support from Norgine and Gilead; Dr. Bass, consulting fees from Genentech and Norgine; and Dr. Chalasani, consulting fees from Gilead, Genentech, Amylin, Pfizer, Advanced Life Sciences, Metabasis (now Ligand Pharmaceuticals), AtheroGenics, Karo Bio, Debiovision, Eli Lilly, Johnson \& Johnson, Teva Pharmaceuticals, Abbott, Norgine, Ockham, and Salix Pharmaceuticals, grant support from Gilead, Eli Lilly, Debiovision, and Monarch LifeSciences, reimbursements for travel and accommodations from Salix Pharmaceuticals, Gilead, Genentech, Advanced Life Sciences, Amylin, Metabasis (now Ligand Pharmaceuticals), AtheroGenics, Johnson \& Johnson, Debiovision and Pfizer, and fees for serving on the data and safety monitoring board for Takeda and being named on patents pending for biomarkers of nonalcoholic steatohepatitis held by Indiana University. No other potential conflict of interest relevant to this article was reported. Disclosure forms provided by the authors are available with the full text of this article at NEJM.org.

APPENDIX

Members of the Nonalcoholic Steatohepatitis Clinical Research Network are as follows. Steering Committee — J. Lavine (cochair), University of California San Diego; A. Sanyal (cochair), Virginia Commonwealth University; S. Abrams, Baylor College of Medicine; N. Bass, University of California San Francisco; N. Chalasani, Indiana University; A.M. Diehl, Duke University; K. Kowdley, Virginia Mason Medical Center; R. Loomba, University of California San Diego; A. McCullough, Case Western Reserve University; J. Molleston, Indiana 
University; K. Murray, University of Washington; P. Robuck, National Institute of Diabetes and Digestive and Kidney Diseases; P. Rosenthal, University of California San Francisco; B. Neuschwander-Tetri, Saint Louis University; J. Tonascia, Johns Hopkins University, Data Coordinating Center. Protocol Subcommittee - N. Chalasani, A. Sanyal, A. Unalp. Clinical centers enrolling subjects in the PIVENS trial - Case Western Reserve University and Cleveland Clinic Foundation - both in Cleveland: A. McCullough, D. Bringman, S. Dasarathy, K. Edwards, C. Hawkins, Y.-C. Liu, N. Rogers, R. Sargent; Duke University Medical Center, Durham, NC: A.M. Diehl, M. Abdelmalek, M. Gottfried, C. Guy, P. Killenberg, S. Kwan, Y.-P. Pan, D. Piercy, M. Smith; Indiana University School of Medicine, Indianapolis: N. Chalasani, P. Bhimalli, O.W. Cummings, A. Klipsch, L. Lee, J. Molleston, L. Ragozzino, R. Vuppalanchi; Saint Louis University, St. Louis: B.A. Neuschwander-Tetri, J. Derdoy, J. Hoffmann, D. King, J. Siegner, S. Stewart, J. Thompson, E. Brunt; University of California San Diego, San Diego: J.E. Lavine, C. Behling, L. Clark, J. Durelle, T. Hassanein, L. Petcharaporn, J.B. Schwimmer, C. Sirlin, T. Stein; University of California San Francisco, San Francisco: N.M. Bass, K. Bambha, L.D. Ferrell, D. Filipowski, R. Merriman, M. Pabst, M. Rosenthal, P. Rosenthal, T. Steel; Virginia Commonwealth University, Richmond: A.J. Sanyal, S. Boyett, M.J. Contos, M. Fuchs, A. Jones, V.A.C. Luketic, B. Sandhu, C. Sargeant, K. Selph, M. White; Virginia Mason Medical Center, Seattle: K.V. Kowdley, G. Gyurkey, J. Mooney, J. Nelson, S. Ackermann, C. Saunders, A. Stead, C. Wang, M. Yeh. Resource centers - National Cancer Institute, Bethesda, MD: D. Kleiner; National Institute of Diabetes and Digestive and Kidney Diseases, Bethesda, MD: E. Doo, J. Everhart, J.H. Hoofnagle, P.R. Robuck (project scientist), L. Seeff; Johns Hopkins University Bloomberg School of Public Health (Data Coordinating Center), Baltimore: J. Tonascia, P. Belt, F. Brancati, J. Clark, R. Colvin, M. Donithan, M. Green, M. Isaacson, W. Kim, L. Miriel, A. Sternberg, A. Ünalp, M. Van Natta, I. Vaughn, L. Wilson, K. Yates.

\section{REFERENCES}

1. Browning JD, Szczepaniak LS, Dobbins R, et al. Prevalence of hepatic steatosis in an urban population in the United States: impact of ethnicity. Hepatology 2004;40:1387-95.

2. Ludwig J, Viggiano TR, McGill DB, Oh BJ. Nonalcoholic steatohepatitis: Mayo Clinic experiences with a hitherto unnamed disease. Mayo Clin Proc 1980;55:434-8.

3. Adams LA, Sanderson S, Lindor KD, Angulo P. The histological course of nonalcoholic fatty liver disease: a longitudinal study of 103 patients with sequential liver biopsies. J Hepatol 2005;42:132-8.

4. Ekstedt M, Franzén LE, Mathiesen UL, et al. Long-term follow-up of patients with NAFLD and elevated liver enzymes. Hepatology 2006;44:865-73.

5. Marchesini G, Brizi M, Bianchi G, et al. Nonalcoholic fatty liver disease: a feature of the metabolic syndrome. Diabetes 2001;50:1844-50.

6. Sanyal AJ, Campbell-Sargent C, Mir shahi F, et al. Nonalcoholic steatohepatitis: association of insulin resistance and mitochondrial abnormalities. Gastroenterology 2001;120:1183-92.

7. McClain CJ, Mokshagundam SP, Barve SS, et al. Mechanisms of non-alcoholic steatohepatitis. Alcohol 2004;34:67-79.

8. Belfort R, Harrison SA, Brown K, et al. A placebo-controlled trial of pioglitazone in subjects with nonalcoholic steatohepatitis. N Engl J Med 2006;355:2297-307. 9. Promrat K, Lutchman G, Uwaifo GI, et al. A pilot study of pioglitazone treatment for nonalcoholic steatohepatitis. Hepatology 2004;39:188-96.

10. Caldwell SH, Hespenheide EE, Redick JA, Iezzoni JC, Battle EH, Sheppard BL. A pilot study of a thiazolidinedione, troglitazone, in nonalcoholic steatohepatitis. Am J Gastroenterol 2001;96:519-25.

11. Neuschwander-Tetri BA, Brunt EM, Wehmeier KR, Oliver D, Bacon BR. Improved nonalcoholic steatohepatitis after
48 weeks of treatment with the PPARgamma ligand rosiglitazone. Hepatology 2003;38:1008-17.

12. Sanyal AJ, Mofrad PS, Contos MJ, et al. A pilot study of vitamin E versus vitamin $\mathrm{E}$ and pioglitazone for the treatment of nonalcoholic steatohepatitis. Clin Gastroenterol Hepatol 2004;2:1107-15.

13. Lavine JE. Vitamin E treatment of nonalcoholic steatohepatitis in children: a pilot study. J Pediatr 2000;136:734-8.

14. Harrison SA, Torgerson S, Hayashi P, Ward J, Schenker S. Vitamin E and vitamin $\mathrm{C}$ treatment improves fibrosis in patients with nonalcoholic steatohepatitis. Am J Gastroenterol 2003;98:2485-90.

15. Ratziu V, Giral P, Jacqueminet S, et al. Rosiglitazone for nonalcoholic steatohepatitis: one-year results of the randomized placebo-controlled Fatty Liver Improvement with Rosiglitazone Therapy (FLIRT) Trial. Gastroenterology 2008;135:100-10.

16. Chalasani NP, Sanyal AJ, Kowdley KV, et al. Pioglitazone versus vitamin $\mathrm{E}$ versus placebo for the treatment of non-diabetic patients with non-alcoholic steatohepatitis: PIVENS trial design. Contemp Clin Trials 2008;30:88-96.

17. Kleiner DE, Brunt EM, Van Natta M, et al. Design and validation of a histological scoring system for nonalcoholic fatty liver disease. Hepatology 2005;41:1313-21. 18. Skinner HA, Holt S, Schuller R, Roy J, Israel Y. Identification of alcohol abuse using laboratory tests and a history of trauma. Ann Intern Med 1984;101:847-51. 19. Conigrave KM, Hall WD, Saunders JB. The AUDIT questionnaire: choosing a cutoff score: Alcohol Use Disorder Identification Test. Addiction 1995;90:1349-56. 20. Powell EE, Cooksley WG, Hanson R, Searle J, Halliday JW, Powell LW. The natural history of nonalcoholic steatohepatitis: a follow-up study of forty-two patients for up to 21 years. Hepatology 1990;11:7480
21. Bacon BR, Farahvash MJ, Janney CG, Neuschwander-Tetri BA. Nonalcoholic steatohepatitis: an expanded clinical entity. Gastroenterology 1994;107:1103-9.

22. Younossi ZM, Gramlich T, Liu YC, et al. Nonalcoholic fatty liver disease: assessment of variability in pathologic interpretations. Mod Pathol 1998;11:560-5.

23. Ratziu V, Charlotte F, Heurtier A, et al. Sampling variability of liver biopsy in nonalcoholic fatty liver disease. Gastroenterology 2005;128:1898-906.

24. Chalasani N, Wilson L, Kleiner DE, Cummings OW, Brunt EM, Unalp A. Relationship of steatosis grade and zonal location to histological features of steatohepatitis in adult patients with non-alcoholic fatty liver disease. J Hepatol 2008;48:82934.

25. Hampton T. Diabetes drugs tied to fractures in women. JAMA 2007;297:1645. 26. Brunt EM, Kleiner DE, Wilson LA, et al. Portal chronic inflammation in nonalcoholic fatty liver disease (NAFLD): a histologic marker of advanced NAFLDclinicopathologic correlations from the Nonalcoholic Steatohepatitis Clinical Research Network. Hepatology 2009;49: 809-20.

27. Lutchman G, Modi A, Kleiner DE, et al. The effects of discontinuing pioglitazone in patients with nonalcoholic steatohepatitis. Hepatology 2007;46:424-9.

28. Mannucci E, Monami M, Lamanna C, Gensini GF, Marchionni N. Pioglitazone and cardiovascular risk: a comprehensive meta-analysis of randomized clinical trials. Diabetes Obes Metab 2008;10:1221-38. 29. Miller ER III, Pastor-Barriuso R, Dalal D, Riemersma RA, Appel LJ, Guallar E. Meta-analysis: high-dosage vitamin E supplementation may increase all-cause mortality. Ann Intern Med 2005;142:37-46. Copyright (c) 2010 Massachusetts Medical Society. 